\title{
Malignant peripheral nerve sheath tumours in magnetic resonance imaging: primary and recurrent tumour appearance, post-treatment changes, and metastases
}

\author{
Sam Sedaghat ${ }^{A, B, C, D, E, F}$, Frederick Schmitz ${ }^{A, B, C, D, E, F}$, Martin Grözinger ${ }^{A, B, C, D, E, F}$, Maya Sedaghat ${ }^{A, B, C, D, E, F}$ \\ Institute for Diagnostic and Interventional Radiology and Nuclear Medicine, BG University Hospital Bergmannsheil, Bochum, Germany
}

\begin{abstract}
Purpose: To analyse the appearance of primary and recurrent malignant peripheral nerve sheath tumours (MPNSTs) in magnetic resonance imaging (MRI) with a focus on configuration, and to assess the occurrence of loco-regional post-treatment changes and metastases during post-treatment follow-up.

Material and methods: Twenty patients with histologically proven MPNST underwent post-treatment 1.5 T MRI. Primary and recurrent MPNSTs were examined for configuration, contrast enhancement, extent, and signal intensity in MRI. Loco-regional post-treatment changes and information on metastases were extracted from the follow-up.

Results: MPNSTs occurred most often in the extremities $(p=0.006)$. Twenty per cent $(n=4)$ of the patients developed recurrences, with a total of 24 lesions. Recurrent MPNSTs were significantly smaller than primary MPNSTs $(p=0.003)$. Primary MPNSTs mostly occurred unifocally as multilobulated or ovoid and heterogeneous lesions with mostly well-defined borders. Recurrent MPNSTs purely occurred multifocally as mostly nodular $(p<0.001)$, multilobulated, or ovoid lesions. $80 \%, 65 \%$ and $30 \%$ of the patients showed post-treatment subcutaneous oedema ( $p=0.002$ to 0.03$)$, muscle oedema $(p=0.02)$, and seroma, respectively. Twenty-five per cent $(n=5)$ of patients presented metastases during follow-up. The relative risk in patients with recurrences to develop lung or lymph node metastases is eightfold $(p=0.056)$.

Conclusions: While primary MPNSTs mostly appear unifocally as multilobulated or ovoid lesions, recurrent MPNSTs purely occur multifocally as mostly nodular lesions. Subcutaneous and muscle oedema are very common locoregional post-treatment changes. Patients with recurrences have a higher risk for lung and lymph node metastases.

Key words: recurrence, MRI, metastases, oedema, malignant peripheral nerve sheath tumour, appearance.
\end{abstract}

\section{Introduction}

Malignant peripheral nerve sheath tumours (MPNST) are rare and highly aggressive neoplasms arising from Schwann cells [1,2]. The expected incidence of MPNST is reported to be $0.1 / 100,000$ per year [2,3], and they account $3-10 \%$ of all soft tissue tumours [2-5]. $40-50 \%$ of MPNST are associated with neurofibromatosis type 1 (NF-1) gene mutation [2,6]. In about $50 \%$ of the patients MPNST occur sporadically [7]. MPNST have a high po- tential for local recurrence and a high risk for metastatic spread [6]. They most often appear in the limbs, trunk/ retroperitoneum, and head/neck region of middle-aged to elderly adults [7]. MPNST responds poorly to chemoor radiotherapy; therefore, surgery with wide resection margins is the most comonly performed curative treatment $[1,6,8,9]$. As MPNST are very rare neoplasms, there are very few data regarding imaging of MPNST. Therefore, the aim of this study was to analyse the appearance of MPNSTs in magnetic resonance imaging (MRI) with

Correspondence address:

Sam Sedaghat, Institute for Diagnostic and Interventional Radiology and Nuclear Medicine, BG University Hospital Bergmannsheil, Bochum, Germany,

e-mail: samsedaghat1@gmail.com

Authors' contribution:

A Study design · B Data collection · C Statistical analysis · D Data interpretation - E Manuscript preparation · F Literature search · G Funds collection 
a focus on configuration and to assess the occurrence of loco-regional post-treatment changes and metastases during post-treatment follow-up.

\section{Material and methods}

\section{Patients}

From 248 patients with malignant soft-tissue sarcoma 28 consecutive patients with histologically proven diagnosis of MPNST between 2012 and 2018 were reviewed. Eight patients were excluded due to insufficient imaging data. Four of these patients had no follow-up imaging at our institution and four patients underwent other examinations than MRI. The twenty remaining patients underwent MRI follow-up for a minimum of one year (equivalent to four MRI follow-up scans) at our institution after resection of primary MPNST. The primary and recurrent tumours were radiographically examined for signal intensity, contrast enhancement (intensity, heterogeneous/homogenous), configuration, extent, and limitation in MRI. As general epidemiological data, the age of the patients at primary diagnosis, the localisation of the primary and recurrent tumours, and the recurrence-free MRI follow-up interval were analysed. As loco-regional post-treatment soft-tissue changes, subcutaneous and muscle oedema, post-operative seroma, reactive lymphadenopathy, bone oedema, and synovialitis were included. Additionally, the patients were screened for information about metastases.

Magnetic resonance imaging was performed with a 1.5 Tesla MRI system (MAGNETOM Symphony, Siemens Healthineers). The MRI protocol was performed with the following sequences: axial T2-weighted (TE: 64-114 ms, TR: 3010-5840 ms, FOV: 22-44 cm, SD: 5-6 mm), axial T1-weighted (TE: $10-14 \mathrm{~ms}$, TR: $587-868 \mathrm{~ms}$, FOV: $22-44 \mathrm{~cm}$, $\mathrm{SD}$ : 5-6 mm), axial proton density-weighted (PDw) (TE: 26-36 ms, TR: 2740-4610 ms, FOV: 22-40 cm, SD: 5-6 mm), coronal Turbo-Inversion Recovery-Magnitude (TIRM) (TE: 68-77 ms, TR: $4410-6980 \mathrm{~ms}$, FOV: $37-45 \mathrm{~cm}$, SD: 4-6 mm), axial (1012 ms, TR: 645-865 ms, FOV: $22-44 \mathrm{~cm}$, SD: 5-6 mm), coronal (TE: 10-13 ms, TR: 533-1440 ms, FOV: $37-45 \mathrm{~cm}$, SD: 4-6 mm), and sagittal (TE: $10-13 \mathrm{~ms}$, TR: 577-866 ms, FOV: 22-37 cm, SD: 5-10 mm) T1-weighted after application of IV contrast agent.

Two dedicated sarcoma radiologists with a minimum of five years of experience in sarcoma diagnostics reviewed each MRI with findings reached by consensus.

\section{Statistical analysis}

If not indicated otherwise, data are given as median values with range (minimum to maximum) or mean with standard deviation (SD). Parametric and nonparametric tests to compare group values ( $\chi^{2}$ test, Mann-Whitney $U$ test, ANOVA) were performed as indicated. Specificity, sensitivity, and area under the curve (AUC) were determined using $2 \times 2$ tables. Furthermore, risk ratios (RR) for the determination of relative risks were analysed. Statistical significance for all tests was set at a level of $p<0.05$. Statistical analysis was done using the IBM-SPSS version 22.0 software package (IBM, Armonk, NY, USA).

\section{Ethics approval}

The study was conducted in accordance with, and was approved by, the responsible Ethics Committee.

\section{Results}

The mean age of the patients was 49 years (SD: 19, min.: 16, max.: 81). Recurrences occurred after a mean of 26 months (SD: 23.3, min.: 9, max.: 60). MPNST occurred most often in the axilla (20\%), followed by the thigh (15\%), elbow, and lower leg (10\% each). Altogether MPNST arose significantly most often in the extremities (13 cases; $p=0.006$ ). Recurrent MPNST purely occurred in the axilla, thigh, and hip (Figure 1). In four of 20 patients we found recurrences of MPNST in MRI follow-up (20\%), with 24 lesions altogether. Recurrent MPNST were significantly smaller than primary MPNST in mean diameter $(p=0.003)$. The main appearance of primary MPNST was multilobulated (Figure 2) and ovoid with heterogeneous contrast enhancement and well-defined borders $(p=0.002)$. In one case primary MPNST presented as infiltrative and in another case with a few contrast enhancements (Table 1). All primary MPNSTs presented unifocally. Recurrent MPNSTs purely occurred multifocally as mostly nodular lesions ( $p<0.001$, Figure 3 ) with homogenous contrast enhancement and well-defined borders (Table 2), but also multilobulated/heterogeneous (Figure 4) or ovoid. All primary and recurrent tumours presented with muscle iso-intense T1-weighted and high PD-weighted and TIRM signal. The overall most common loco regional post-treatment changes after resection of MPNST were subcutaneous oedema ( $80 \% ; p=0.002$ to 0.03 ), followed by muscle oedema $(65 \% ; p=0.02)$ and post-operative seroma (30\%; $p=0.04$ ). In patients with the presence of post-operative

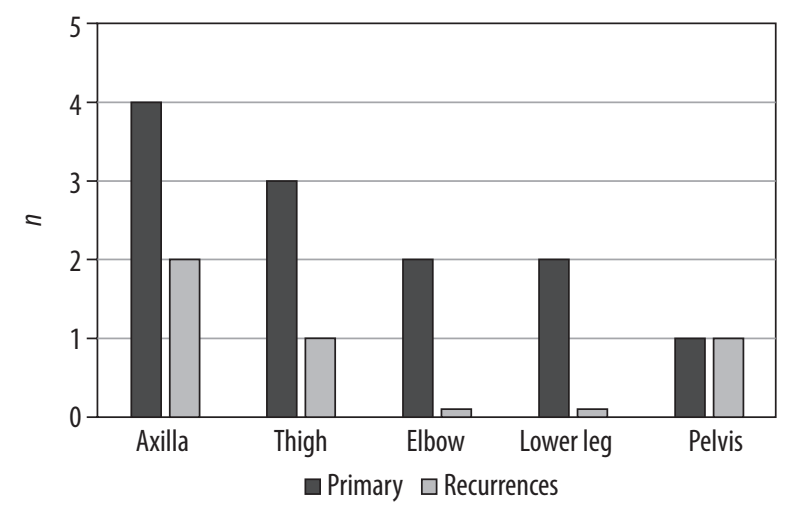

Figure 1. Main localisations of primary and recurrent malignant peripheral nerve sheath tumours, shown as amount of patients $(n)$ 

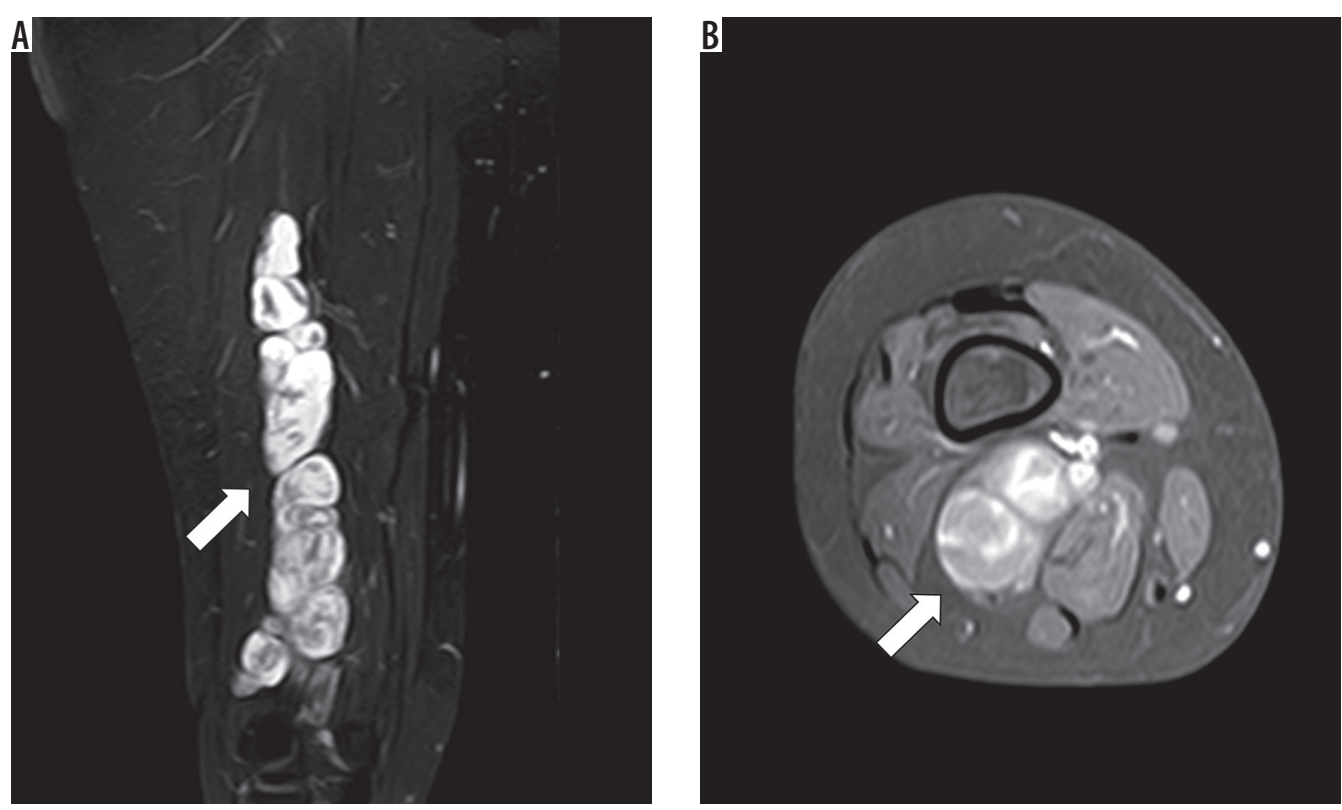

Figure 2. Primary malignant peripheral nerve sheath tumour at 1.5 Tesla magnetic resonance imaging: A) coronary proton density weighted; B) axial T1-weighted with fat saturation after application of IV contrast agent of the lower leg of a 48-year-old female patient. The tumour presents as a multilobulated mass with well-defined borders and heterogenous contrast enhancement (white arrow)

seroma the risk for recurrence was non-significantly higher $(\mathrm{RR}=2$; 95\% CI: 0.55-7.3; $p=0.29$; Table 3$)$.

Metastases were found in five patients (25\%) with three cases of lung and lymph node metastases, respectively, and one case each of pleural, bone, chest wall, and liver metastases. Patients with recurrences had a non-significantly higher risk for lung and lymph node metastases $(\mathrm{RR}=8,95 \%$ CI: 0.955-67.7, $p=0.056$; Table 4$)$.

\section{Discussion}

In this study we mainly analysed the appearance of primary and recurrent MPNST in MRI with a focus on configuration, and assessed the occurrence of loco-regional post-treatment changes and metastases during post-treatment follow-up. Until now there have been only a few publications on imaging of MPNST because MPNST is a very rare disease [10].

The median recurrence-free follow-up interval in our study is 54 months. This has to be distinguished from the overall recurrence-free follow-up interval, which is often higher due to the lack of MRI follow-up data. Actually, an MRI follow-up interval of five years after resection of the primary tumour is intended [11], but it is frequently observed that a lot of patients do not undergo their total follow-up in one and the same radiological department.

Table 1. Magnetic resonance imaging contrast agent behaviour, appearance, and limitation of 8 consecutive primary malignant peripheral nerve sheath tumours (MPNST) with age of manifestation in years and the mean diameter of the primary tumour in millimetres

\begin{tabular}{|c|c|c|c|c|c|c|c|c|}
\hline \multirow{2}{*}{$\begin{array}{l}\text { Primary } \\
\text { MPNST } \\
\text { (No.) } \\
\text { P-primary }\end{array}$} & \multicolumn{3}{|c|}{ Contrast agent enhancement } & \multicolumn{2}{|c|}{ Limitation } & \multirow[t]{2}{*}{ Appearance } & \multirow{2}{*}{$\begin{array}{c}\text { Age of } \\
\text { manifestation } \\
\text { (years) }\end{array}$} & \multirow{2}{*}{$\begin{array}{l}\text { Mean diameter } \\
(\mathrm{mm})\end{array}$} \\
\hline & Homogenous & Heterogenous & Intensity & Sharply & Infiltrative & & & \\
\hline P1 & - & $x$ & +++ & $x$ & - & Ovoid & 56 & 51 \\
\hline P2 & - & $x$ & ++ & $x$ & $x$ & Multilobulated & 81 & 58 \\
\hline P3 & - & $x$ & +++ & $x$ & - & Multilobulated & 35 & 70 \\
\hline P4 & - & $x$ & ++ & $x$ & - & Multilobulated & 22 & 81 \\
\hline P5 & - & $x$ & +++ & $x$ & - & Multilobulated & 48 & 94 \\
\hline P6 & - & $x$ & + & $x$ & - & Ovoid & 74 & 19 \\
\hline P7 & - & $X$ & ++ & $x$ & - & Ovoid & 36 & 37 \\
\hline P8 & - & $x$ & +++ & $x$ & - & Multilobulated & 43 & 67 \\
\hline$p$-value & \multicolumn{2}{|c|}{-} & & \multicolumn{2}{|c|}{0.002} & 0.18 & & \\
\hline Mean, SD & & & & & & & $49.4,20.1$ & $59.6,24.0$ \\
\hline
\end{tabular}



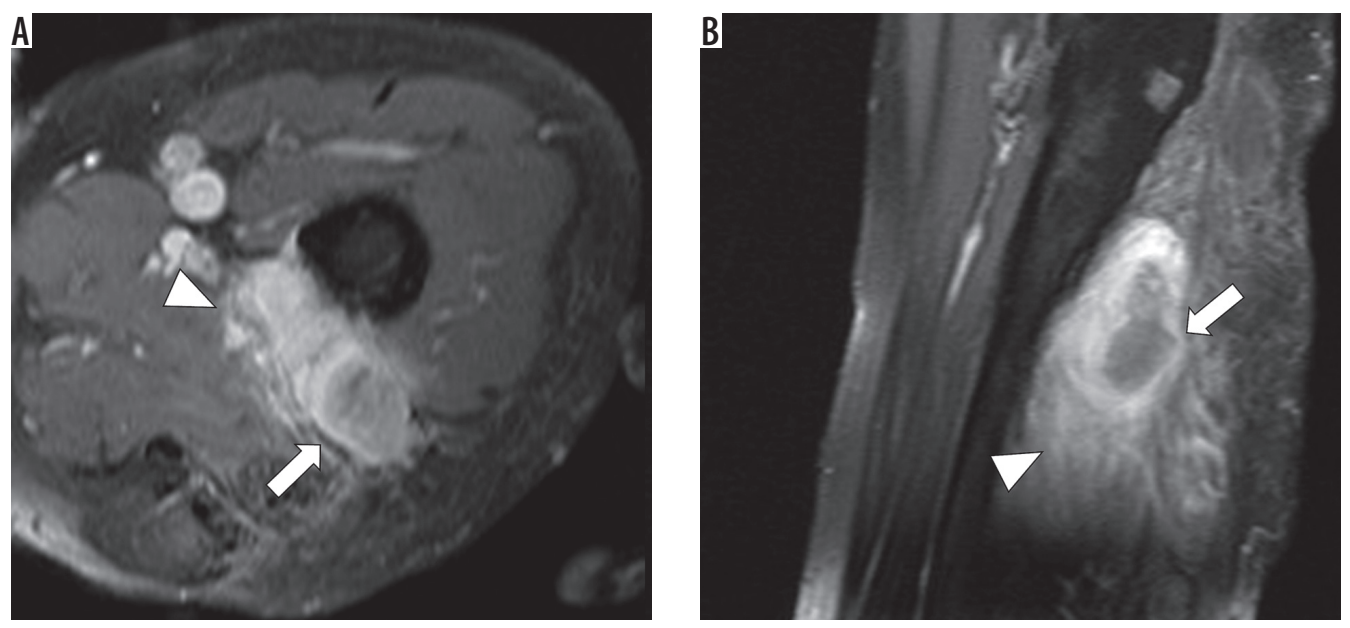

Figure 3. Recurrent malignant peripheral nerve sheath tumour at 1.5 T magnetic resonance imaging of the left thigh of a 57-year-old patient (T1-weighted with fat saturation after application of IV contrast agent) in axial (A) and sagittal (B) view. The tumour mass in the left thigh appears multilobulated with heterogenous contrast enhancement (white arrow) and infiltrative behaviour (white arrow head)

Table 2. Magnetic resonance imaging contrast agent behaviour, appearance and limitation of 4 consecutively cases of recurrent malignant peripheral nerve sheath tumours (MPNST) with altogether 24 lesions. Additionally, the age of manifestation in years and the mean diameter of the primary tumour in millimetres are shown

\begin{tabular}{|c|c|c|c|c|c|c|c|c|}
\hline $\begin{array}{l}\text { Recurrent } \\
\text { MPNST } \\
\text { (No.) }\end{array}$ & \multicolumn{3}{|c|}{ Contrast agent enhancement } & \multicolumn{2}{|c|}{ Limitation } & Appearance & $\begin{array}{c}\text { Age of } \\
\text { manifestation } \\
\text { (years) }\end{array}$ & $\begin{array}{c}\text { Mean } \\
\text { diameter } \\
(\mathrm{mm})\end{array}$ \\
\hline $\mathrm{R}$-recurrence & Homogenous & Heterogenous & Intensity & Sharply & Infiltrative & All multifocal & & \\
\hline \multirow[t]{4}{*}{ R1 } & - & $x$ & ++ & $x$ & $x$ & Multilobulated (2) & 21 & 19 \\
\hline & $x$ & - & ++ & $x$ & - & Nodular (2) & & 4 \\
\hline & $x$ & - & ++ & - & $x$ & Streaky (1) & & 8 \\
\hline & $x$ & - & ++ & $x$ & $x$ & Ovoid (3) & & 11 \\
\hline R2 & $x$ & - & ++ & $x$ & - & Multiple nodular (10) & 52 & 5 \\
\hline \multirow[t]{2}{*}{ R3 } & - & $x$ & ++ & $x$ & $x$ & Multilobulated & 57 & 21 \\
\hline & $x$ & - & ++ & $x$ & - & Nodular (2) & & 6.5 \\
\hline \multirow[t]{3}{*}{ R4 } & - & $x$ & ++ & $x$ & $x$ & Multilobulated (1) & 36 & 14 \\
\hline & - & $x$ & ++ & $x$ & - & Ovoid (1) & & 10 \\
\hline & $x$ & - & ++ & $x$ & - & Nodular (1) & & 5 \\
\hline$p$-value & \multicolumn{2}{|c|}{-} & & \multicolumn{2}{|c|}{-} & Nodular: $p<0.001$ & & \\
\hline Mean, SD & & & & & & & $41.5,16.3$ & - \\
\hline
\end{tabular}
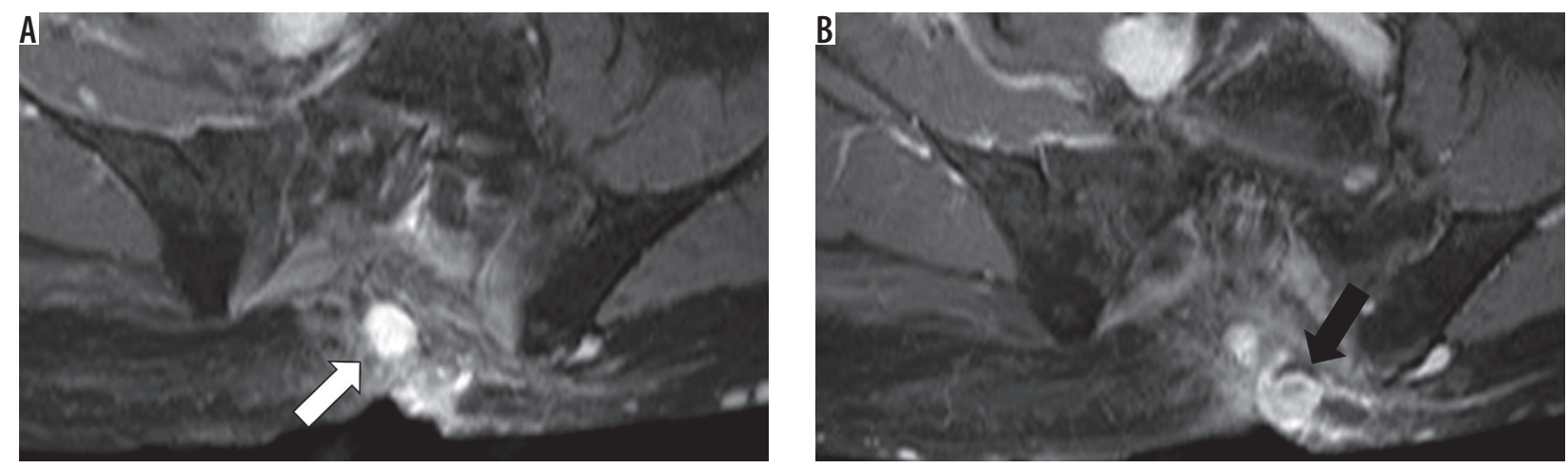

Figure 4. Recurrent malignant peripheral nerve sheath tumour at $1.5 \mathrm{~T}$ magnetic resonance imaging of the pelvis of a 57-year-old patient (T1-weighted with fat saturation after application of IV contrast agent) in axial view. A and B show two nodular lesions next to the sacrum with homogenous contrast enhancement and well-defined borders (white and black arrows) 
Table 3. Post-treatment changes totally and in patients with and without recurrences of malignant peripheral nerve sheath tumours

\begin{tabular}{|c|c|c|c|c|c|c|c|}
\hline $\begin{array}{l}\text { Post-treatment } \\
\text { changes }\end{array}$ & $\begin{array}{c}\text { Total } \\
\text { (20 patients) } \\
n(\%)\end{array}$ & \multicolumn{3}{|c|}{$p$-value } & $\begin{array}{c}\text { Patients with } \\
\text { recurrence } \\
\text { (4 patients) } \\
n(\%)\end{array}$ & $\begin{array}{l}\text { Patients without } \\
\text { recurrence } \\
\text { (16 patients) } \\
n(\%)\end{array}$ & $\begin{array}{c}\text { Relative } \\
\text { risk ratio (RR); } \\
p \text {-value }\end{array}$ \\
\hline Subcutaneous tissue oedema & $16(80)$ & \multirow{3}{*}{0.002} & \multirow{3}{*}{0.03} & & $3(75)$ & $13(81)$ & - \\
\hline Muscle oedema & $13(65)$ & & & \multirow{2}{*}{0.02} & $2(50)$ & $11(68)$ & - \\
\hline Post-operative seroma & $6(30)$ & & & & $2(50)$ & $4(25)$ & $\mathrm{RR}=8 ; p=0.056$ \\
\hline Lymphadenopathy & $4(20)$ & & & & - & $4(25)$ & - \\
\hline Bone oedema & $1(5)$ & & & & - & $1(6)$ & - \\
\hline Muscle abscess & $1(5)$ & & & & $1(25)$ & - & - \\
\hline Synovialitis & $1(5)$ & & & & - & $1(6)$ & - \\
\hline
\end{tabular}

Statistical significance: $p<0.05$

Table 4. Metastases occurred within magnetic resonance imaging follow-up in total and in patients with and without recurrences. The relative risk ratio was determined for patients with/without recurrence regarding lung and lymph node metastases

\begin{tabular}{|l|c|c|c|c|} 
Metastases & $\begin{array}{c}\text { Total } \\
\text { (20 patients) } \\
n(\%)\end{array}$ & $\begin{array}{c}\text { Patients with recurrence } \\
(4 \text { patients) } \\
n(\%)\end{array}$ & $\begin{array}{c}\text { Patients without recurrence } \\
\text { (16 patients) } \\
n(\%)\end{array}$ & $\begin{array}{c}\text { Relative risk ratio (RR); } \\
p \text {-value }\end{array}$ \\
\hline Lung & $3(15)$ & $2(50)$ & $1(6)$ & $8 ; 0.056$ \\
\hline Lymph node & $3(15)$ & $2(50)$ & $1(6)$ & $8 ; 0.056$ \\
\hline Bone & $1(5)$ & $1(25)$ & - & - \\
\hline Pleura & $1(5)$ & $1(25)$ & - & - \\
\hline Chest wall & $1(5)$ & $1(25)$ & $1(6)$ & - \\
\hline Liver & $1(5)$ & - & & - \\
\hline
\end{tabular}

MPNST is reported to be mostly located in the extremities and the trunk but may also occur in other sites like the head and neck $[12,13]$. In our study the extremities were the primary site of MPNST as well, followed by the axilla.

Magnetic resonance imaging is the favoured imaging modality for pre- and post-treatment evaluation of softtissue tumours [14]. The application of contrast agent is not required in all cases, but contrast enhancement may help to distinguish between post-treatment changes and the recurrent tumour $[14,15]$. Nevertheless, the specificity of MRI for the detection of recurrences of soft-tissue tumours is reported to be moderate [16]. In contrast, Park C. et al. described a high sensitivity and specificity of MRI for the detection of recurrent soft-tissue sarcoma (90\% and $97.7 \%$, respectively) [17]. Twenty per cent of our patients developed local recurrences within MRI follow-up, with 24 lesions altogether. Local recurrences are reported to occur in $10-50 \%$ of all soft-tissue sarcomas [18] and in 35\% of patients with MPNSTs [2], after resection of the primary tumours.

In our study recurrent MPNSTs presented significantly smaller than the primary tumours. A reason for this may be the earlier detection of the recurrent tumours within the MRI follow-up scans, which are routinely performed at intervals of 3-12 months. The mean diameter of primary MPNST is reported to range from 1 to $20 \mathrm{~cm}$ (median size of $8-12 \mathrm{~cm})[5,19,20]$.
Previous studies mainly assess MRI features of primary MPNST with a focus on MRI signal characteristics. In this study we mainly focused on the MRI appearance of both primary and recurrent MPNST. MPNST presents with high signal intensity in T2 STIR and PD FS and low signal intensity in T1 sequences [5,19,21]. Furthermore, MPNST is reported to appear in different shapes with mostly heterogeneous appearance and well-defined margins $[5,19,20]$. MPNST may also present as ovoid with uniform enhancement $[19,20]$. In our study we found two main appearances of primary MPNSTs: multilobulated and ovoid, with both heterogeneous/marked contrast enhancement and well-defined borders. In only one case primary MPNST presented with infiltrative and in another case with only a few contrast enhancements. Against this, recurrent MPNSTs purely occurred multifocal as mostly nodular lesions. As a result, radiologists should look for a minimum of a second lesion after detection of any recurrent MPNST lesion within MRI follow-up.

As mentioned, MRI plays an important role in the postoperative evaluation of soft-tissue changes. Differentiation between post-treatment soft-tissue changes and tumour recurrences has to be made, which is often problematic [22]. It is reported that post-treatment bone and soft-tissue changes are often apparent on MRI [18]. In our study $80 \%$ of the patients present with subcutaneous oe- 
dema, followed by muscle oedema $(65 \%)$ and post-operative seroma (30\%). Post-operative seromas are reported to occur in approximately $17-19 \%$ of cases after resection of soft-tissue sarcomas [22-24]. In addition, post-operative seromas normally appear homogenous and well-defined with rim contrast enhancement [18]. Furthermore, 37\% of patients with soft-tissue tumours may present with bone marrow oedema [25]. In our study we detected one patient with bone marrow oedema after resection of primary MPNST.

Previous data describe that $39 \%$ of the patients with MPNST present with distant metastasis, mainly to the lung (28\%), but also to the bone (5\%), liver (2\%), and lymph nodes (1\%) [2]. Lung metastases occur in about $20 \%$ of patients diagnosed with soft-tissue sarcoma [26]. We found metastases in five patients, with three cases of lung and lymph node metastases, respectively. Especially patients with recurrence have a non-significantly higher risk for lung and lymph node metastases.

\section{Conclusions}

While primary MPNSTs appear unifocally as multilobulated or ovoid and heterogeneous lesions, recurrent MPNSTs purely occur multifocally as mainly nodular lesions. Therefore, radiologists should look for a minimum of a second lesion after detection of any recurrent MPNST lesion. Posttreatment subcutaneous and muscle oedema are common occurrences in MRI follow-up. Patients with recurrences have a higher risk for lung and lymph node metastases.

\section{Conflict of interest}

The authors report no conflict of interest.

\section{References}

1. Friedrich RE, Kluwe LAN, Fünsterer C, et al. Malignant peripheral nerve sheath tumors (MPNST) in neurofibromatosis type 1 (NF1): diagnostic findings on magnetic resonance images and mutation analysis of the NF1 gene. Anticancer Res 2005; 25: 1699-1702.

2. Anghileri M, Miceli R, Fiore M, et al. Malignant peripheral nerve sheath tumors: prognostic factors and survival in a series of patients treated at a single institution. Cancer 2006; 107: 1065-1074.

3. Enzinger FM, Weiss SW. Epithelioid smooth muscle tumors. In: Soft tissue tumors. $3^{\text {rd }}$ ed. CV Mosby 1995: 511-22.

4. Grobmyer SR, Reith JD, Shahlaee A, et al. Malignant peripheral nerve sheath tumor: molecular pathogenesis and current management considerations. J Surg Oncol 2008; 97: 340-349.

5. Wasa J, Nishida Y, Tsukushi S, et al. MRI features in the differentiation of malignant peripheral nerve sheath tumors and neurofibromas. Am J Roentgenol 2010; 194: 1568-1574.

6. Watson KL, Al Sannaa GA, Kivlin CM, et al. Patterns of recurrence and survival in sporadic, neurofibromatosis type 1 - associated, and radiation-associated malignant peripheral nerve sheath tumors. J Neurosurg 2017; 126: 319-329.

7. Schaefer IM, Fletcher CDM, Hornick JL. Loss of H3K27 trimethylation distinguishes malignant peripheral nerve sheath tumors from histologic mimics. Mod Pathol 2015; 29: 3-14.

8. Wong WW, Hirose T, Scheithauer BW, et al. Malignant peripheral nerve sheath tumor: analysis of treatment outcome. Int J Radiat Oncol Biol Phys 1998; 42: 351-360.

9. Bradford D, Kim A. Malignant peripheral nerve sheath tumor: analysis of treatment outcome. Curr Treat Options Oncol 2015; 16: 12.

10. Sperandio M, Di Poce I, Ricci A, et al. Malignant peripheral nerve sheath tumour: CT and MRI findings. Case Rep Radiol 2013; 2013: 517879.

11. Aga P, Singh R, Parihar A, et al. Imaging spectrum in soft tissue sarcomas. Indian J Surg Oncol 2011; 2: 271-279.

12. Hirose T, Scheithauer BW, Sano T. Perineurial malignant peripheral nerve sheath tumor (MPNST): a clinicopathologic, immunohistochemical, and ultrastructural study of seven cases. Am J Surg Pathol 1998; 22: 1368-1378.

13. Stucky C-CH, Johnson KN, Gray RJ et al. Malignant peripheral nerve sheath tumors (MPNST): the Mayo Clinic experience. Ann Surg Oncol 2012; 19: 878-885.

14. Garner HW, Kransdorf MJ, Bancroft LW, et al. Benign and malignant soft-tissue tumors: posttreatment MR imaging. Radiographics 2009; 29: 119-134.

15. Kransdorf MJ, Murphey MD. Imaging of soft tissue tumors. Lippincott Williams \& Wilkins, Philadelphia 2006.

16. Del Grande F, Subhawong T, Weber K, et al. Detection of soft-tissue sarcoma recurrence: added value of functional MR imaging techniques at 3.0 T. Radiology 2014; 271: 499-511.

17. Park S-Y, Chung HW, Chae SY, et al. Comparison of MRI and PET$\mathrm{CT}$ in detecting the loco-regional recurrence of soft tissue sarcomas during surveillance. Skeletal Radiol 2016; 45: 1375-1384.

18. Fain AD, Beaman FD. Magnetic resonance imaging of soft tissue masses. Semin Roentgenol 2017; 52: 227-240.

19. Kragha KO. Malignant peripheral nerve sheath tumor: MRI and CT findings. Case Rep Radiol 2015; 2015: 241259.

20. Li CS, Huang GS, Wu HD, et al. Differentiation of soft tissue benign and malignant peripheral nerve sheath tumors with magnetic resonance imaging. Clin Imaging 2008; 32: 121-127.

21. Lin J, Martel W. Cross-sectional imaging of peripheral nerve sheath tumors: characteristic signs on CT, MR imaging, and sonography. Am J Roentgenol 2001; 176: 75-82.

22. James SLJ, Davies AM. Post-operative imaging of soft tissue sarcomas. Cancer Imaging 2008; 8: 8-18.

23. Poon-Chue A, Menendez L, Gerstner MM, et al. MRI evaluation of post-operative seromas in extremity soft tissue sarcomas. Skeletal Radiol 1999; 28: 279-282.

24. Skibber JM, Lotze MT, Seipp CA, et al. Limb-sparing surgery for soft tissue sarcomas: wound related morbidity in patients undergoing wide local excision. Surgery 1987; 102: 447-452.

25. Hwang S, Lefkowitz R, Landa J, et al. Local changes in bone marrow at MRI after treatment of extremity soft tissue sarcoma. Skeletal Radiol 2009; 38: 11-19.

26. Digesu CS, Wiesel O, Vaporciyan AA, et al. Management of sarcoma metastases to the lung. Surg Oncol Clin N Am 2016; 25: 721-733. 